\title{
Photoisomerization of $\beta$-lonone Protonated Schiff Base in the Gas Phase
}

Neville J. A. Coughlan, Claire M. Wallace, Brian D. Adamson, and Evan J.

$$
\text { Bieske* }
$$

School of Chemistry, The University of Melbourne, Victoria 3010, Australia

E-mail: evanjb@unimelb.edu.au

Phone: +613 8344 7082. Fax: +61393475180 


\begin{abstract}
The photoisomerization of $\beta$-ionone protonated Schiff base (BIPSB) is investigated in the gas phase by irradiating mobility-selected ions in a tandem ion mobility spectrometer with tunable radiation. Four distinguishable isomers are produced by electrospray ionization whose structures are deduced from their collision cross sections and photoisomerization behaviour along with density functional theory calculations. They include two geometric isomers of BIPSB with trans or cis configurations about the polyene chain's terminal $\mathrm{C}=\mathrm{N}$ double bond, a bicyclic structure formed through electrocyclization of the polyene chain, and a Z-retro- $\gamma$-ionone isomer. Although trans BIPSB and 9-cis BIPSB have similar photoisomerization action spectra, with a maximum response at $375 \mathrm{~nm}$, they photoconvert to different isomers. The trans BIPSB isomer transforms to the bicyclic form upon exposure to light over the 320-400 nm range, whereas the cis BIPSB isomer is prevented by steric hindrance from forming the bicyclic BIPSB isomer following irradiation and is proposed instead to form the 7,9-dicis isomer. Neither the bicyclic isomer nor the Z-retro- $\gamma$-ionone isomer respond strongly to near-UV light.
\end{abstract}

\title{
1 Introduction
}

$\beta$-ionone is a small terpene, consisting of an ionone ring attached to a short polyene chain. It is commercially important in vitamin A manufacture, is a common decomposition product of larger polyenes, including $\beta$-carotene, ${ }^{1}$ and, because of its woody, violet odour, is a component of natural and confected scents. ${ }^{2}$ As shown in Fig. 1 , the presence of alternating single and double bonds means that $\beta$-ionone can adopt different cis-trans conformations and that it can undergo photochemical transformations akin to those occurring for longer chain polyenes, including retinal and $\beta$-carotene. ${ }^{3}$ In solution, UV radiation converts trans $\beta$-ionone to 7 -cis $\beta$-ionone, which exists in equilibrium with the $\alpha$-pyran isomer. ${ }^{4-9}$ At the same time, the Z-retro- $\gamma$-ionone isomer is formed slowly and irreversibly through a 
$[1,5]$ sigmatropic $\mathrm{H}$ atom shift. ${ }^{9}$

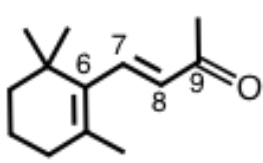

$\beta$-ionone

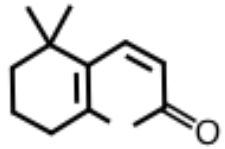

$\beta$-ionone 7-cis

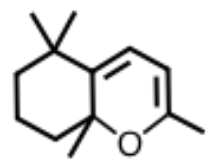

a-pyran

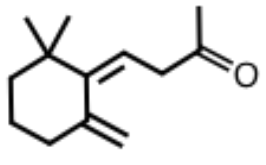

Z-retro- $\gamma$-ionone

Figure 1: $\beta$-ionone and its isomers.

Recently, we have explored photo-induced structural rearrangements of molecular ions in the gas phase, using ion mobility mass spectrometry as a structural probe. One focus of the work has been retinal protonated Schiff base (RPSB), which undergoes photoisomerization about $\mathrm{C}=\mathrm{C}$ bonds in the polyene chain. At higher laser powers it was found that RPSB decomposes to generate the $\beta$-ionone protonated Schiff base [BIPSB, Fig. 2] in a process involving electrocylization of the polyene chain and elimination of a toluene molecule. ${ }^{10}$ By separating the ions in a drift tube ion mobility spectrometer (IMS) it was shown that there are at least 3 isomers in the gas phase, which may include the trans [Fig. 2(i)], 7-cis [Fig. 2(ii)], and 9-cis BIPSB [Fig. 2(iv)] isomers, the bicyclic isomer [Fig. 2(v)], or the retro- $\gamma$-ionone protonated Schiff base [GIPSB, Fig. 2(vi)]. In this study we have investigated the different BIPSB isomers, following their photo-induced structural rearrangements using a tandem IMS-laser-IMS spectrometer. The experimental strategy involves selection of one isomer in the first IMS stage followed by laser excitation, and separation of product photoisomers in a second IMS stage. To help interpret the gasphase measurements we have conducted density functional theory (DFT) calculations for minimum energy structures and isomerization barriers.

An advantage of the ion mobility approach is that the structural and photochemical properties of the ions are characterized free from solvent interactions, providing experimental data that can be compared directly with theoretical predictions for the isolated molecules. We have deployed the same strategy to explore photoisomerization of 
(i)<smiles>[2H][N+]=C(C)C=CC1=C(C)CCCC1(C)C</smiles>

BIPSB<smiles>[R][NH+]=C/C=C/C1=C(C)CCCC1(C)C</smiles>

BIPSB 9-cis (ii)<smiles>CC(=[NH+])/C=C\C1=C(C)CCCC1(C)C</smiles>

(v)<smiles>[2H][N+]1([2H])C(C)=CC=C2C(C)(C)CCCC21C</smiles>

bicyclic (iii)<smiles>[R][NH+]=C(C)/C=C/C1=C(C)CCCC1(C)C</smiles><smiles>C=C1CCCC(C)(C)C1=CCC(C)=[NH+]</smiles>

GIPSB

Figure 2: Low energy structures of $\beta$-ionone protonated Schiff base (BIPSB) and the bicyclic and $\gamma$-ionone protonated Schiff base (GIPSB) isomers.

carbocyanine dyes, ${ }^{11-13}$ retinal protonated Schiff base, ${ }^{14,15}$ and the protonated spiropyran/merocyanine photoswitch. ${ }^{16}$

In analogy to the situation for RPSB, BIPSB is expected to isomerize around the $C_{7}=C_{8}$ double bond in the polyene chain following excitation to the $S_{1}$ state, converting trans BIPSB to 7-cis BIPSB [Fig. 2(ii)]. Earlier studies of $\beta$-ionone in solution have implicated the 7-cis isomer as a gateway structure for electrocyclization to form the bicyclic isomer (Fig. 1) ${ }^{7}$ If a similar situation prevails for BIPSB in the gas phase, one might expect that the electrocyclization efficiency will depend critically on the configuration of the terminal $\mathrm{C}=\mathrm{N}$ bond (9-trans versus 9-cis), due to steric hindrance presented by the $\mathrm{R}$ group ( $n$-butyl in this study). Tandem ion mobility spectrometry is ideal to explore such steric effects because the target isomer can be selected in the first ion mobility stage with separation of the photoisomers in the second stage. 


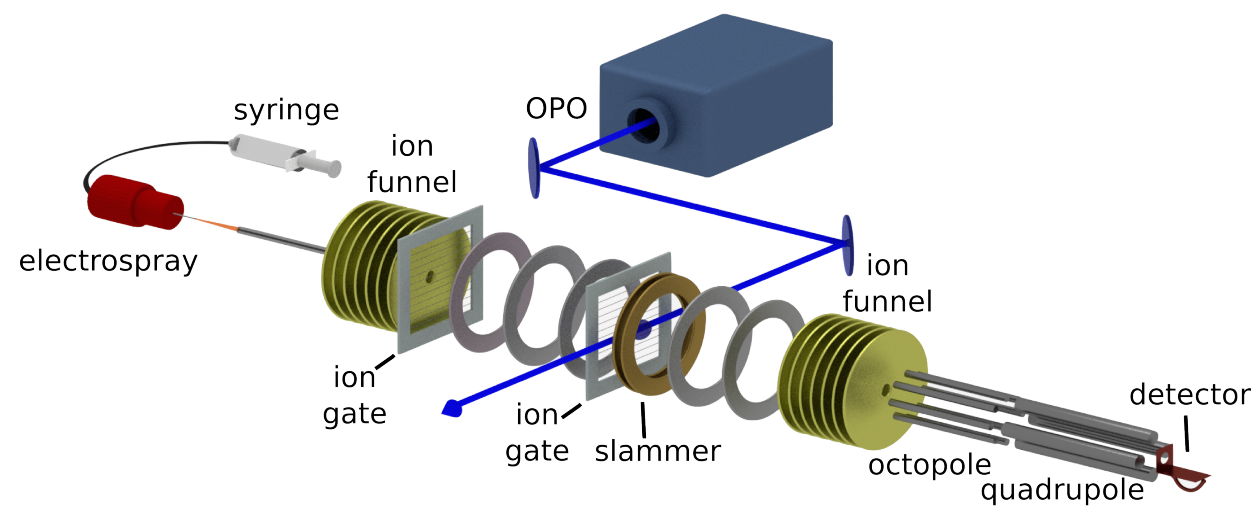

Figure 3: Tandem ion mobility machine used for investigating photoisomerization of $\beta$-ionone protonated Schiff base. See refs. 13 and 16 for more details.

\section{Experimental Methods}

The experimental setup has been described previously, ${ }^{13,16}$ and involves combining ion mobility spectrometry and laser spectroscopy using the purpose-built machine illustrated schematically in Fig. 3. BIPSB was synthesized as outlined in ref. 10. BIPSB ions produced through electrospray ionization were collected by an ion funnel (IF1) and injected through an electrostatic gate into a drift-tube ion mobility mass spectrometer in which the various isomers were separated spatially and temporally according to their collision cross section with low pressure buffer gas ( $\mathrm{N}_{2}$ gas, $\mathrm{P}=6.4$ Torr). After traversing the drift region, the ions were collected by a second ion funnel (IF2) and passed through a $3 \mathrm{~mm}$ orifice into an octopole ion guide $\left(\mathrm{P}=2 \times 10^{-4}\right.$ torr $)$ and then through a second $5 \mathrm{~mm}$ orifice and into a quadrupole mass filter tuned to $m / z=248$ (the mass of BIPSB). The ions were detected by a channeltron whose output was passed to a discriminator and then to a multichannel scaler to generate an arrival time distribution (ATD). Typically, the mobility resolution of the instrument, defined as the arrival time divided by the peak width, is 70-80.

Approximately half-way through the drift region the ions encountered an electrostatic ion gate that could be opened to select a particular isomer that was then irradiated with the output of a tunable, pulsed optical parametric oscillator (OPO, 10 ns pulse width). Resulting photoisomer ions were separated from the parent ions in the second IMS stage. 
The apparatus was operated in laser on/off mode with alternate ion packets in the instrument being overlapped with a light pulse from the OPO. The difference in the laser-on and laser-off ATDs reflects the effect of light on the drifting ions. For the light fluxes used in the study ( $\leq 0.5 \mathrm{~mJ} / \mathrm{cm}^{2} /$ pulse), photodissociation was a negligible process. Photoisomerization action (PISA) spectra were measured by scanning the laser wavelength and recording the photoisomerization yield. Collision cross sections $(\Omega)$ were measured by varying the drift voltage $\left(V_{d}\right)$ across the second IMS stage, while monitoring the arrival time $\left(t_{a}\right)$. A plot of $t_{a}$ versus $1 / V_{d}$ yields a straight line whose slope is proportional to the collision cross section. ${ }^{15}$

\section{Results}

Arrival time distributions of BIPSB generated by electrospray ionization under different conditions are shown in Fig. 4. Under low collision energy conditions, with the drive voltage to the first ion funnel switched off, the ATD was dominated by two peaks, A and B, with A being slightly more intense than B [see Fig 4(a)]. Applying a RF drive voltage to IF1 energized the ions before they were injected into the drift tube, leading to peak A becoming more intense than $\mathrm{B}$, and the appearance of a new faster peak $\mathrm{C}$, along with unresolved signal between peaks B and C [see Fig 4(b)]. Exposure of the BIPSB solution in the syringe to UV light $(350-400 \mathrm{~nm})$ increased the intensity of peak $C$ relative to peaks $A$ and B and also lead to the appearance of a fourth peak (D) between B and C [see Fig 4(c)]. Collision cross sections for the observed isomers are compiled in Table 1.

In order to connect measured collision cross sections with particular isomers, equilibrium structures, energies and collision cross sections were calculated for the 22 most stable geometric BIPSB isomers using density functional theory (DFT) with the M06-2X functional and cc-pVDZ basis set. These calculations were performed with the Gaussian 09 suite. ${ }^{17}$ Due to steric hindrance some of these isomers are not stable or lie very high 


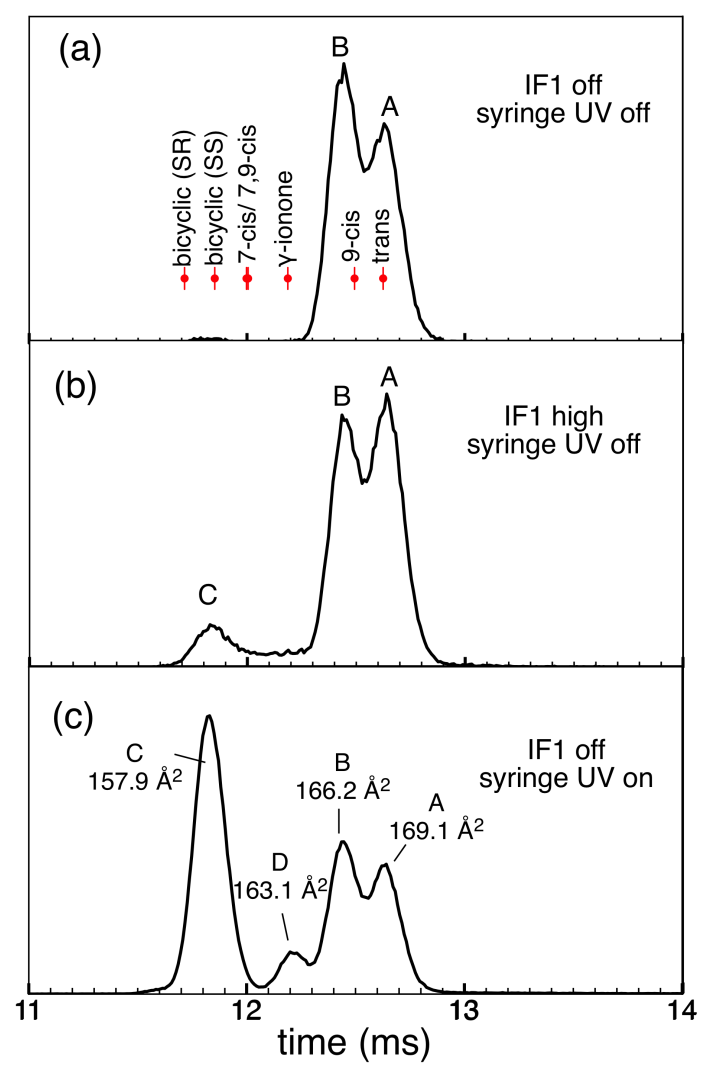

Figure 4: Arrival time distributions for $\beta$-ionone with (a) no UV and no RF drive voltage to IF1, (b) no UV and RF drive voltage to IF1 set high, (c) UV irradiation of BIPSB solution for 90 minutes prior to electrospray and no RF drive voltage to IF1. Predicted arrival times of trans, 9-cis, 7-cis, 7,9-dicis, bicyclic and $\gamma$-ionone isomers are shown in (a). Measured collision cross sections for isomers A-D are given in (c)

in energy. Similar calculations were performed for the bicyclic and $\gamma$-ionone isomers [Fig. 2(v) and (vi)]. Collision cross sections were determined using the trajectory method in Mobcal ${ }^{18,19}$ with collision parameters taken from ref. 20. Energies and collision cross sections for the various isomers are compiled in the SI.

As for retinal protonated Schiff base, BIPSB has 3 possible configurations for the $6 \mathrm{~s}$ bond [6s-cis(+), 6s-cis(-) and 6s-trans], ${ }^{14,21}$ and two possible configurations for each of the other bonds in the polyene chain, giving 24 possible geometric isomers. The calculations show that the 9-cis isomers are the lowest energy structures with the trans and 7-cis isomers lying $3-7 \mathrm{~kJ} / \mathrm{mol}$ and $13-19 \mathrm{~kJ} / \mathrm{mol}$ higher in energy, respectively. The bicyclic isomers have similar energies to the 7-cis isomers. 


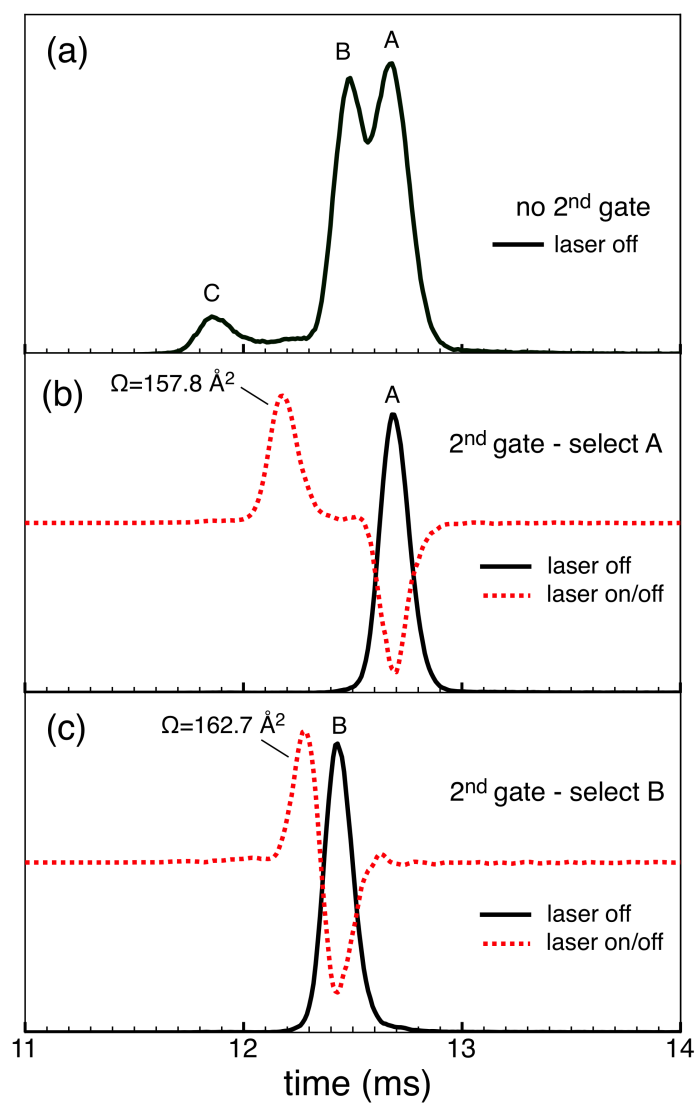

Figure 5: (a) Arrival time distributions for $\beta$-ionone. Measured cross sections for peaks A-C are indicated. (b) ATD with selection of peak A by IG2, and difference in laser-on and laser-off ATDs. (c) ATD with selection of peak B by IG2, and difference in laser-on and laser-off ATDs.

Barriers for rotation around the $6 \mathrm{~s}$ and 8 s single bonds are predicted through DFT calculations to be relatively low $(\leq 40 \mathrm{~kJ} / \mathrm{mol})$ so that there should be rapid interconversion between the 6s-cis(+), 6s-cis(-) and 6s-trans conformers and the 8s-trans and 8s-cis conformers as the ions pass through the drift region. Barriers for isomerization around the $C_{7}=C_{8}$ and $\mathrm{C}_{9}=\mathrm{N}$ double bonds are much higher and are calculated to exceed $120 \mathrm{~kJ} / \mathrm{mol}$. Under these circumstances one might expect to observe four different BIPSB isomer families corresponding to trans, 7-cis, 9-cis and 7,9-dicis isomers. Effective energies and collision cross sections for these 4 isomer families were calculated as a Boltzmann average over the different contributing $6 \mathrm{~s}$ and 8 s isomers, as described in refs. 14 and 15 , and the SI. Collision cross sections calculated in this way are slightly larger than the measured cross 
sections (by $\approx 2 \%$ ). However, as shown in Fig. $4(\mathrm{a})$, when uniformly scaled so that the slowest peak (A) corresponds to the trans isomer, the second slowest peak (B) lines up with the 9-cis isomer. These two isomers are predicted to be the most stable forms of BIPSB. The less stable 7-cis and 7,9-dicis isomers are predicted to have similar cross sections and line up with the unresolved signal between peaks B and C in Fig. 4(b). The good correspondence between measured and predicted collision cross sections is illustrated in Fig. S.5.

The bicyclic form [Fig. 2(v)] has 2 distinguishable families of stereoisomers, SS and $R R$, and $R S$ and $S R$, according to the configuration at the two stereo centres. The $S S$ and $R R$ isomers are calculated to lie $14.7 \mathrm{~kJ} / \mathrm{mol}$ above 9-cis BIPSB and have a collision cross section $\Omega_{\text {calc }}=162.5 \AA^{2}$, whereas the $R S$ and $S R$ isomers lie $22.7 \mathrm{~kJ} / \mathrm{mol}$ above 9-cis BIPSB and have a collision cross section $\Omega_{\text {calc }}=160.6 \AA^{2}$. Predicted arrival times for these isomers correspond to peak C in the ATD [see Fig. 4(a)]. The $\gamma$-ionone isomer [GIPSB, Fig. 2(vi)] is calculated to lie somewhat higher in energy ( $31.0 \mathrm{~kJ} / \mathrm{mol}$ above 9-cis BIPSB) and is predicted to have a collision cross section $\Omega_{\text {calc }}=166.2 \AA^{2}$, consistent with its assignment as isomer D. The ATD peak assignments based on calculated energies and collision cross sections are summarised in Table 1.

Table 1: Measured collision cross sections $\left(\Omega_{m}\right)$, measured maximum absorption wavelengths, and calculated collision cross sections $\left(\bar{\Omega}_{\text {calc }}\right)$ for BIPSB isomers. Assignments and measured collision cross sections for the photoisomers are also given. For measured collision cross sections, estimated absolute errors are $\pm 5 \AA^{2}$ and relative errors are $\pm 0.3 \AA^{2}$. Representative structures are shown in Fig. 2, while DFT optimised structures are provided in the SI.

\begin{tabular}{ccccccc}
\hline peak & assignment & $\begin{array}{c}\Omega_{m} \\
\left(\AA^{2}\right)\end{array}$ & $\begin{array}{c}\bar{\Omega}_{\text {calc }} \\
\left(\AA^{2}\right)\end{array}$ & $\begin{array}{c}\lambda_{\max } \\
(\mathrm{nm})\end{array}$ & $\begin{array}{c}\text { photoisomer } \\
\text { assignment }\end{array}$ & $\begin{array}{c}\text { photoisomer } \Omega_{m} \\
\left(\AA^{2}\right)\end{array}$ \\
\hline A & trans BIPSB & 169.1 & 173.1 & 375 & bicyclic & 157.8 \\
B & 9-cis BIPSB & 166.2 & 171.3 & 375 & $\gamma$-ionone or 7,9-dicis & 162.7 \\
C & bicyclic & 157.9 & $162.7^{\dagger}$ & - & - & - \\
D & $\gamma$-ionone & 163.1 & $166.2 \ddagger$ & - & - & - \\
\hline \multicolumn{6}{c}{ ' Cross section for the lowest energy S,S (chair) isomer } \\
‡ Cross section for the lowest energy 6-cis (chair) isomer
\end{tabular}

The identities of the four observed isomers are confirmed by their responses to light. 


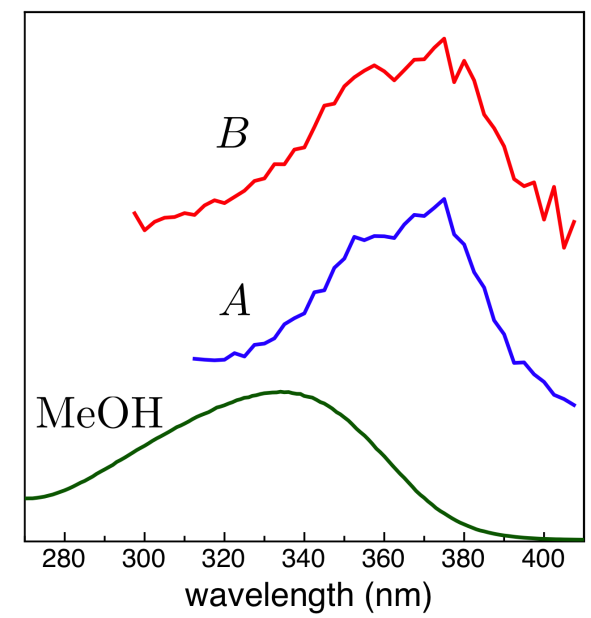

Figure 6: Photoisomerization action spectra of 9-trans (A) and 9-cis (B) BIPSB measured by monitoring photoisomer formation. The measured absorption spectrum of BIPSB in methanol solution is shown at bottom.

When exposed to near-UV light (300-400 nm) mid-way through the drift region of the IMS, isomers A and B converted to more compact isomers. As shown in Fig. 5, isomer A photoconverts to an isomer with a collision cross section of $157.8 \AA^{2}$, almost certainly isomer $\mathrm{C}\left(\Omega_{m}=157.9 \AA^{2}\right)$. The photoisomer formed from isomer $\mathrm{B}$ has a cross section of $162.7 \AA^{2}$, very close to the cross section of isomer $\mathrm{D}\left(\Omega_{m}=163.1 \AA^{2}\right)$, consistent with it being either GIPSB or 7,9-dicis BIPSB. Photoisomerization action (PISA) spectra for isomers A and $\mathrm{B}$, obtained by plotting the photoisomer yield as a function of wavelength, are shown in Fig. 6. The PISA spectra for A and B are very similar, with both possessing a maximum at $375 \mathrm{~nm}$, suggesting that both isomers have similar conjugated structures, consistent with their assignments as the trans and 9-cis isomers [Fig. 2(i) and (iv)] .

We were unable to photoisomerize isomers $C$ and $D$ with light over the $300-400 \mathrm{~nm}$ range. This is consistent with their assignments as the bicyclic and GIPSB isomers [Fig. 2(v) and (vi)], both of which should absorb shorter wavelength light $(\leq 300 \mathrm{~nm})$ due to their lack of conjugation. Assignments of peak $C$ to the bicyclic isomer and peak $D$ to the GIPSB isomer are consistent with their formation kinetics. In agreement with previous observations, ${ }^{22}$ we found that the bicyclic isomer is generated more rapidly in an irradiated 
solution of $\beta$-ionone than the $\gamma$-ionone form, but that $\gamma$-ionone dominates at longer times.

If isomers A and B are indeed the trans and 9-cis isomers and have similar photoisomerization action spectra why do they produce different photoproducts (the bicyclic isomer and the 7,9-dicis BIPSB or GIPSB isomers, respectively)? The isomer specificity can be rationalized through the mechanism shown in Fig. 7, whereby the trans BIPSB isomer is photoconverted by UV light into 7-cis BIPSB, which, in turn, undergoes rapid electrocyclization to form the compact bicyclic structure shown in Fig. 2(v), corresponding to isomer C. For the mechanism to be viable, photoexcitation must sequentially convert the trans isomer to the 7-cis isomer, then to the 7,8s-cis isomer, and finally to the bicyclic structure over a barrier. Calculations at the DFT M06-2X/cc-pVDZ level for a minimal model in which the butyl group is replaced by a methyl group, suggest that the barrier for the final step is $\approx 130 \mathrm{~kJ} / \mathrm{mol}$ above the 7-cis isomer (see SI). In contrast, although photoexcitation can convert the 9-cis isomer to the 7,9-dicis isomer, steric interference between the bulky $n$-butyl group and the ionone ring is likely to prevent electrocyclization and formation of the bicyclic structure. A similar explanation was advanced by Ramamurthy et al. to rationalize preferential formation of bicyclic products from the 9-trans isomer of $\beta$-ionylideneacetonitrile compared to the 9-cis isomer for which steric interference prevents cyclization. ${ }^{7}$ Other assignments for the ATD peaks and the photochemical mechanism are less convincing. For example, it is improbable that B corresponds to the 7-cis isomer, because in that case it would most probably photoconvert to the all-trans isomer (i.e. isomer A).

As explained above, isomer $\mathrm{D}$ has a collision cross section and photophysical behaviour that are consistent with its assignment as GIPSB. To computationally explore the energetics for the [1-5] sigmatropic $\mathrm{H}$ atom shift that converts trans BIPSB to GIPSB, we used a minimal model with the butyl group replaced by a methyl group, finding that the barrier is $+139 \mathrm{~kJ} / \mathrm{mol}$ (see SI). The magnitude of this barrier is similar to that for the formation of the bicyclic isomer. 


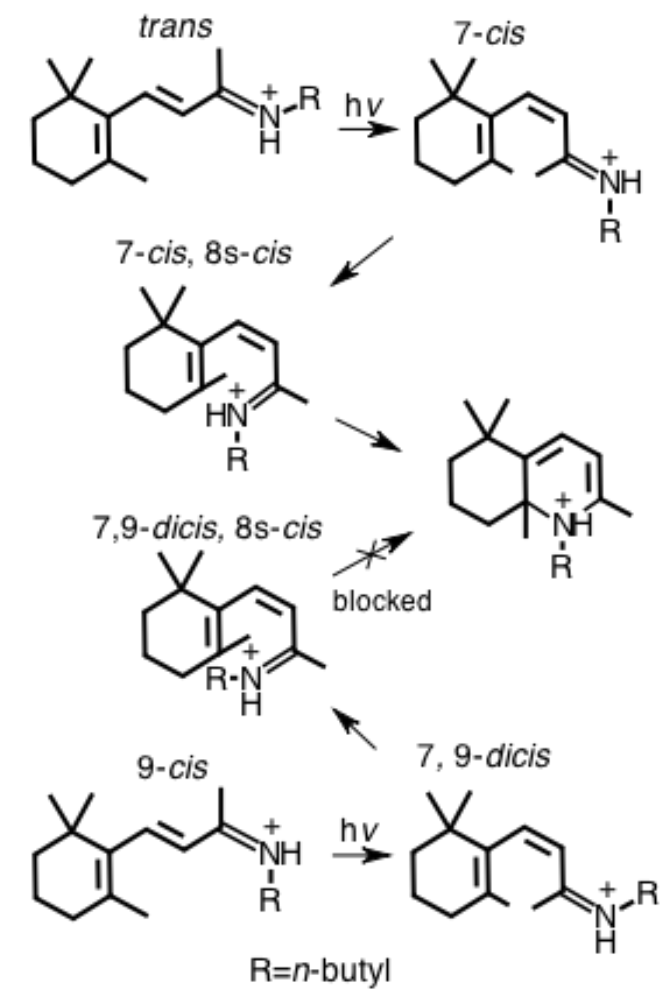

Figure 7: Proposed mechanism for photo-induced cyclization of BIPSB in the gas phase.

\section{Discussion}

The experimental and theoretical data provide a new view of the structural properties and photochemical behaviour of BIPSB and its isomers in the gas-phase. The ion mobility measurements show that when BIPSB is electrosprayed into the gas phase at least 4 isomers are formed. If the solution is protected from light and the ions are treated gently, the drifting ion population contains mainly trans and 9-cis BIPSB isomers. However, UV irradiation of the electrosprayed solution or collisional excitation in the first ion funnel produces, in addition, the bicyclic and $\gamma$-ionone isomers. The data indicate that in the gas phase, light over the $300-400 \mathrm{~nm}$ range converts trans BIPSB to the bicyclic isomer [Fig. 2(v)], whereas 9-cis BIPSB is photoconverted to 7,9-dicis BIPSB.

Several aspects deserve further comment. First, the observation that the 9-trans and 9-cis isomers (isomers $\mathrm{A}$ and $\mathrm{B}$ ) follow different photoisomerization pathways leading to 
distinct products implies that structural reorganization of BIPSB does not occur through a statistical exploration of the $S_{0}$ potential energy surface following internal conversion from the $S_{1}$ state, otherwise one would expect that following photon absorption the 9-trans and 9-cis isomers would scramble to form a similar set of product isomers. Rather, it seems likely that photoexcitation is followed by torsion about the $C_{7}=C_{8}$ bond on the $S_{1}$ potential energy surface (PES) leading to a conical intersection that transfers the system to the $\mathrm{S}_{0}$ PES in either the 7-trans or 7-cis configuration. Subsequent isomerization around the $8 \mathrm{~s}$ bond converts the 7-cis isomer to an appropriate configuration for electrocyclization to form the bicyclic structure [Fig. 2(v)]. Buffer gas collisions, which occur at a rate of $10^{8}-10^{9} \mathrm{~s}^{-1}$, presumably rapidly remove excess vibrational energy from the nascent photoisomer. The initial photoisomerization steps in this proposed mechanism, which involve torsional deformation on the $S_{1}$ PES leading to a conical intersection, are akin to those responsible for the $c i s \rightarrow$ trans photoisomerization of retinal in rhodopsin proteins ${ }^{23-25}$ and in solution, ${ }^{26}$ and have also been postulated to occur for retinal protonated Schiff base in the gas phase. ${ }^{15}$ However, evidence for generation of specific photoproducts that depend on the starting isomer is much clearer for BIPSB than for RPSB in the gas phase.

\section{Conclusions}

In summary, we have shown that $\beta$-ionone protonated Schiff base can be converted to at least four isomeric forms, which on the basis of their collision cross sections and photoisomerization behaviour in the gas phase, are assigned as the trans, 9-cis, bicyclic and $\gamma$-ionone isomers. In the gas phase, absorption of near-UV light $(320-400 \mathrm{~nm})$ converts trans BIPSB first to the 7-cis BIPSB isomer and then to a compact bicyclic structure, that is unresponsive to visible and near-UV light. The other predominant form, the 9-cis isomer, is converted by $320-400 \mathrm{~nm}$ light to 7,9-dicis BIPSB, but is prevented through steric hindrance from cyclization. The $\gamma$-ionone isomer, which is generated after prolonged irradiation of 
the $\beta$-ionone solution and is formed from trans BIPSB through a [1-5] sigmatropic $\mathrm{H}$ atom shift, is also unresponsive to visible and near-UV light, consistent with the disruption in its conjugation.

\section{Supporting Information}

Contains depictions of the calculated minimum energy structures for $\beta$-ionone isomers along with their energies and collision cross sections, a description of the method for determining calculated collision cross sections, a plot of calculated collision cross sections against measured collision cross sections, and an example of a fitted arrival time distribution for $\beta$-ionone isomers.

\section{Acknowledgement}

This research was supported under the Australian Research Council's Discovery Project funding scheme (Project Numbers DP110100312 and DP120100100).

\section{References}

(1) LaRoe, E.; Shipley, P. Whiskey Composition: Formation of Alpha- and Beta-Ionone by the Thermal Decomposition of Beta-Carotene. J. Agric. Food Chem. 1970, 18, 174-175.

(2) Kraft, P.; Bajgrowicz, J.; Denis, C.; Fráter, G. Odds and Trends: Recent Developments in the Chemistry of Odorants. Angew. Chemie. Int. Ed. 2000, 39, 2980-3010.

(3) Byers, J. Isolation and Identification of the Polyenes Formed During the Thermal Degradation of $\beta, \beta$-Carotene. J. Org. Chem. 1983, 48, 1515-1522.

(4) Mayo, P.; Stothers, J. B.; Yip, R. W. The Irradation of $\beta$-Ionone. Can. J. Chem. 1961, 39, 2135. 
(5) Marvell, E. N.; Caple, G.; Gosink, T. A.; Zimmer, G. Valence Isomerization of a cis-Dienone to an $\alpha$-Pyran. J. Am. Chem. Soc. 1966, 88, 619-620.

(6) Marvell, E. N.; Chadwick, T.; Caple, G.; Gosink, T.; Zimmer, G. Rates of Electrocyclic Reactions. Conversion of $\alpha$-Pyrans to Cis-Dienones. J. Org. Chem. 1972, 37, 2992-2997.

(7) Ramamurthy, V.; Liu, R. S. H. Sigmatropic Hydrogen Migration and Electrocyclization Processes in Compounds in the Vitamin A Series. Photochemistry of Polyenes. X. J. Org. Chem. 1976, 41, 1862-1867.

(8) Ramamurthy, V.; Liu, R. S. H. Photochemistry of Polyenes. IX. Excitation, Relaxation, and Deactivation of Dienes, Trienes, and Higher Polyenes in the Vitamin A Series in the Sensitized Isomerization Reaction. J. Am. Chem. Soc. 1976, 98, 2935-2942.

(9) Kaczor, A.; Reva, I.; Fausto, R. Influence of Cage Confinement on the Photochemistry of Matrix-Isolated E- $\beta$-Ionone: FT-IR and DFT Study. J. Phys. Chem. A 2013, 117, 888-897.

(10) Coughlan, N. J. A.; Adamson, B. D.; Catani, K. J.; Wille, U.; Bieske, E. J. Ion Mobility Unlocks the Photofragmentation Mechanism of Retinal Protonated Schiff Base. J. Phys. Chem. Lett. 2014, 5, 3195-3199.

(11) Adamson, B. D.; Coughlan, N. J. A.; Continetti, R. E.; Bieske, E. J. Changing the Shape of Molecular Ions: Photoisomerization Action Spectroscopy in the Gas Phase. Phys. Chem. Chem. Phys. 2013, 15, 9540-9548.

(12) Adamson, B. D.; Coughlan, N. J. A.; da Silva, G.; Bieske, E. J. Photoisomerization Action Spectroscopy of the Carbocyanine Dye DTC ${ }^{+}$in the Gas Phase. J. Phys. Chem. A 2013, 117, 13319-13325.

(13) Adamson, B. D.; Coughlan, N. J. A.; Markworth, P. B.; Continetti, R. E.; Bieske, E. J. An Ion Mobility Mass spectrometer for Investigating Photoisomerization and Photodissociation of Molecular Ions. Rev. Sci. Instr. 2014, 85, 123109.

(14) Coughlan, N. J. A.; Catani, K. J.; Adamson, B. D.; Wille, U.; Bieske, E. J. Photoisomerization Action Spectrum of Retinal Protonated Schiff Base in the Gas Phase. J. Chem. 
Phys. 2014, 140, 164307.

(15) Coughlan, N. J. A.; Adamson, B. D.; Gamon, L.; Catani, K.; Bieske, E. J. Retinal Shows its True Colours: Photoisomerization Action Spectra of Mobility-Selected Isomers of the Retinal Protonated Schiff Base. Phys. Chem. Chem. Phys. 2015, 17, 22623-22631.

(16) Markworth, P. B.; Coughlan, N. J. A.; Adamson, B. D.; Goerigk, L.; Bieske, E. J. Photoisomerization Action Spectroscopy: Flicking the Protonated Merocyanine-Spiropyran Switch in the Gas Phase. Phys. Chem. Chem. Phys. 2015, 17, 25676-25688.

(17) Frisch, M. J.; Trucks, G. W.; Schlegel, H. B.; Scuseria, G. E.; Robb, M. A.; Cheeseman, J. R.; Scalmani, G.; Barone, V.; Mennucci, B.; Petersson, G. A. et al. Gaussian 09 Revision A.1. Gaussian Inc. Wallingford CT 2009.

(18) Mesleh, M.; Hunter, J.; Shvartsburg, A.; Schatz, G.; Jarrold, M. Structural Information from Ion Mobility Measurements: Effects of the Long-Range Potential. J. Phys. Chem. 1996, 100, 16082-16086.

(19) Shvartsburg, A. A.; Jarrold, M. F. An Exact Hard-Spheres Scattering Model for the Mobilities of Polyatomic Ions. Chem. Phys. Lett. 1996, 261, 86-91.

(20) Campuzano, I.; Bush, M. F.; Robinson, C. V.; Beaumont, C.; Richardson, K.; Kim, H.; Kim, H. I. Structural Characterization of Drug-Like Compounds by Ion Mobility Mass Spectrometry: Comparison of Theoretical and Experimentally Derived Nitrogen Collision Cross Sections. Anal. Chem. 2012, 84, 1026-1033.

(21) Valsson, O.; Filippi, C. Gas-Phase Retinal Spectroscopy: Temperature Effects Are But a Mirage. J. Phys. Chem. Lett. 2012, 3, 908-912.

(22) van Wageningen, A.; Cerfontain, H.; Geenevasen, J. A. J. Photochemistry of NonConjugated Dienones. Part V. Photolysis of (E)- $\beta$-Ionone and its Isomeric $\alpha$-Pyran. J. Chem. Soc., Perkin Trans. II 1975, 1283-1286.

(23) Ernst, O. P.; Lodowski, D. T.; Elstner, M.; Hegemann, P. Microbial and Animal Rhodopsins: Structures, Functions, and Molecular Mechanisms. Chem. Rev. 2013, $114,126-163$. 
(24) Rivalta, I.; Nenov, A.; Garavelli, M. Modelling Retinal Chromophores Photoisomerization: from Minimal Models in Vacuo to Ultimate Bidimensional Spectroscopy in Rhodopsins. Phys. Chem. Chem. Phys. 2014, 16, 16865-16879.

(25) Johnson, P. J. M.; Halpin, A.; Morizumi, T.; Prokhorenko, V. I.; Ernst, O. P.; Miller, R. J. D. Local Vibrational Coherences Drive the Primary Photochemistry of Vision. Nat. Chem. 2015, 7, 980-986.

(26) Bassolino, G.; Sovdat, T.; Soares Duarte, A.; Lim, J. M.; Schnedermann, C.; Liebel, M.; Odell, B.; Claridge, T. D. W.; Fletcher, S. P.; Kukura, P. Barrierless Photoisomerization of 11-cis Retinal Protonated Schiff Base in Solution. J. Am. Chem. Soc. 2015, 137, 1243412437. 


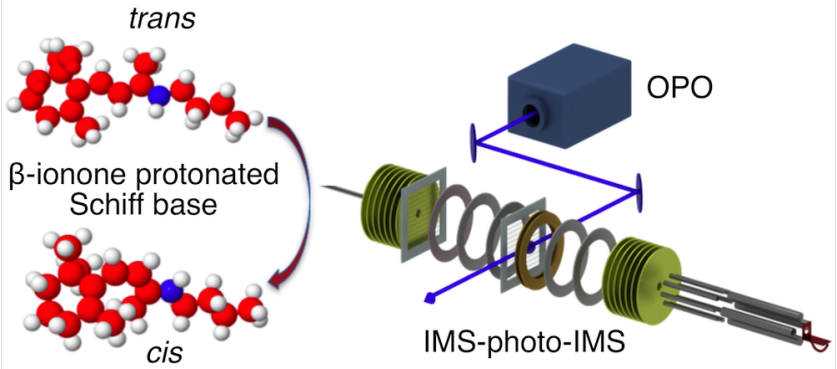

Figure : TOC graphic 


\section{University Library}

\section{- M M N E R VA A gateway to Melbourne's research publications}

Minerva Access is the Institutional Repository of The University of Melbourne

Author/s:

Coughlan, NJA;Wallace, CM;Adamson, BD;Bieske, EJ

Title:

Photoisomerization of beta-Ionone Protonated Schiff Base in the Gas Phase

Date:

2016-08-25

Citation:

Coughlan, N. J. A., Wallace, C. M., Adamson, B. D. \& Bieske, E. J. (2016).

Photoisomerization of beta-Ionone Protonated Schiff Base in the Gas Phase. JOURNAL OF PHYSICAL CHEMISTRY A, 120 (33), pp.6557-6562. https://doi.org/10.1021/ acs.jpca.6b05645.

Persistent Link:

http://hdl.handle.net/11343/123633 